\title{
The Intertextual Influence of Textual Levels in Chinese College Students' English Writing
}

\author{
Hongxia Deng \\ Hunan University, Changsha, Hunan, 410000
}

Keywords: English writing; college students; inter-textual influence

\begin{abstract}
By examining the non-English-like features caused by cross-linguistic differences on discourse level in students' ESL writings, this paper argues that cross-linguistic influence is an non-negligible factor in the teaching and learning of L2 writing. The pedagogical implication of the study is that English teachers should try to arouse students' awareness of social cultural aspects of English language and pay special attention to instill a thorough understanding of western writing convention into language learners so as to improve their writing competence.
\end{abstract}

\section{Introduction}

Writing is a complex and highly intensive, mental, physical and emotional activity. Producing a coherent, fluent and extended piece of writing is a very difficult thing to do in language learning. "It is not uncommon to observe that, while everyone is an expert at speaking their first language, expert at writing is attained only rarely and only with great effort” (Johnson, 2005, p.128). Even native speakers may never master this skill, let alone the nonnative speakers who are also expected to acquire an additional lexical and grammatical knowledge and probably have to go through the painful change of a new writing convention or even thinking pattern in the new language. There are many factors affecting the improvement of foreign language learners's second language writing. Mother tongue influence is one of the most important factors which should not be neglected, especially in Chinese English learning environment.

\section{Comparison on Thesis Statement}

In both Chinese and English languages, a text need a purpose which is either to explain a problem or elaborate a concept, and this purpose is called thesis. However, whether the thesis will take a form of a sentence and where it will appear in a text demonstrate huge differences in Chinese and English.

Given that discourse is a social activity which involves both the writer and the reader, Hinds came up with a distinction in languages based on thesis development: writer-responsible pattern and reader-responsible pattern. When clarifying the reader-responsible pattern, he says the following words:

"It is not the responsibility of the speaker [or the writer] to communicate his message in a clear and direct way. It is the responsibility of the listener or reader to understand what the speaker or the writer intended. In Japanese this apparently requires people to be able to read between lines." Sharing similar culture origin with the Japanese, Chinese tends to be a reader-responsible language in thesis development as well where the purpose of a text or the main idea of a writer cannot be directly seen through an overt statement. It is the reader's role to understand on his/her own through the description in the article. In contrast, English text is not but a writer-responsible language, which means it is the writer's responsibility to clarify the purpose of a text and ambiguity is absolutely forbidden by English norm.

\section{Comparison on the Discourse Pattern}

There are also other versions of discourse structure for this genre of English writing, but nearly 
all of them are of the same nature as the above one. A general opinion (thesis) is stated at the beginning. Then several supporting paragraphs containing detailed information directly related to the general opinion are used to further develop it. Finally, the main idea is summarized in the end paragraph. Cai explains that this is the prominent feature of a general-particular pattern, a deductive line of logic, and he provides a good example to illustrate the pattern [1].

Yet, there are advantages to the underground life, too, and something to be said for imagining whole cities, even mankind generally moving to downward; of having the outermost mile of the Earth's crust hineycombed with passages and structures, like a gigantic ant hill. First, weather would be no longer be important, since it is primarily a phenomenon of atmosphere. Rain, snow, sleet, fog would not trouble the underground world.... Second, local time would no longer be important. On the surface, the tyranny of day and night can not be avoid, and when it is morning in one place, it is noon in aanother... Third, the ecological structure could be stabilized. To a certain extent, mankind encumbers the Earth....

However, relevant studies show that the oriental writing does not follow the above straight-line development (general-particular). Chinese writers tend to suggest rather than state their opinions. Concluded by Cai that Chinese writingsare distinguishable with two features: undulation in plotting, and circular back to the point after an interruption. It is common to see some seemingly off-topic sentences injected into a modern Chinese text, which from the westerners' point of view are nothing but distraction. Kaplan contributes the features to the strictly structured eight-leg essay in Chinese ancient history and others believe it is the more recent rhetoric model of qi-cheng-zhuan-he that is responsible for this kind of phenomenon. "qi" means the opening of a topic in an essay; "cheng" is the preliminary discussion of the topic; in the part of "zhuan" a subtopic which is indirectly related to the topic is introduced and developed; and "he" is the summary of the whole essay [2].

\section{Findings of Cross-linguistic Influence in Chinese Students' ESL Writing on Discourse Level.}

As we can see from the above, in the total 51 sample essays, the percentage of passages with no thesis statement or only presenting the statement in the end takes up more than $40 \%(\approx 13.72 \%+$ $25.49 \%$ ), which is a little bit different from the result obtained by Caiwho finds out that these two cases of thesis statement presenting amount to $60 \%$. The diversity can be explained by the difference in subjects studied. While essays in the present study were all written by English majors, sample essays in his study were collected from students either or not receive professional English training. However, the result in the present research does not mean that Chinese students are less influenced by their native language as distinct results are obtained across three sample groups. If we categorize the two cases of statement presenting (in the end or no thesis statement at all) as the evidence of cross-linguistic influence from Chinese to English, we see the freshmen are mostly affected by Chinese in their ESL writings as they received the least training in English writing theory and practice and are with the lowest language proficiency. The result differences between grades are displayed as below [3]:

As we can see from the above, more than $60 \%$ of freshmen tend to either embed their thesis or purpose in the argumentation without giving a clear statement or only arrive at the thesis as a conclusion in the end, which as we discussed above, can be attributed to the influence by the Chinese writing convention or thinking pattern. And as the grade increases, there is an obvious tendency that more students would like to clearly state their thesis in the beginning and less students would imply their thesis throughout the passage. In other words, the phenomenon of non-English-like feature on thesis statement presenting in Chinese students' ESL writing is less distinguishable as English proficiency increases. More than 85\% juniors prefer to come up with a thesis statement just at the beginning of the essay, comparing with sophomores (64.70\%) and freshmen (29.41\%). This can be explained from the combined action of language transfer and developmental factors. It is reasonable to argue that students in higher grades (juniors in this case) possess higher language proficiency and receive more discipline in English writing, thus they will resort less to the procedureknowledge already acquired in their mother tongue (writing convention or thinking pattern) and probably get used to the deductive way of typical English writing. In 
contrast, students in lower grades (freshmen) will use every strategy available to accomplish the writing task, especially those in their native language.

In order to better explore the influence of Chinese on ESL writing from the perspective of thesis statement presentation, please see the following two examples. The first one is an example for essays of no thesis statement. It begins with extracts of daily conversations about relationships between parents and children. Then the writer introduces the topic of generation gap after the lead-in conversations, and uses the main paragraph talking about the possible reasons, in which he/she even mentions "culture shock" as an analogy to show there are actually huge differences between the two generations. Finally he/she provides a cartoon example to imply that the possible solution may be a role-play. However, there is not a clear statement throughout the whole passage showing the thesis of this argument [4].

The other is a typical ending model of those with thesis statement as a conclusion in the end. The title of this essay is "Honesty is not always the best police", in which the writer first comes up with examples to convince readers that sometime humans have to tell some good-intended lies either to avoid hurting others' feelings or maintain a good interpersonal relationship and then in the last paragraph he/she comes up with the conclusion that "honesty is not always the best police as circumstances change, our police should also change”.

Thus we come to a tentative conclusion that even for English majors who have received professional English training, the cross-linguistic influence is very great in terms of discourse pattern. However, from the author's point of view the mixed type and implicit type of discourse pattern can not be treated as general non-English-like features, as they are resulting from totally different reasons. The implicit type of discourse dates back to the ancient Chinese "suggestive" writing convention which advocates "rambling physically but not spiritually". That's why we usually cannot find the direct logical relations between paragraphs. Whereas the mixed type of discourse pattern is a result of unsuccessful English writing discipline. Within the author's knowledge and from her personal experience, most English writing class (in senior high or college) would spend large amount of time in training students for language matter, such as telling them the formulaic models of discourse development (i.e. first, second...last but not least) or asking them to recite the common transitional words. Actually this method rather pays off in exams as the features students were imposed to remember are the key points to impress judges so as to get higher marks. However, both teachers and students do not pay enough attention to delve deeper to understand the fundamental differences between Chinese and English language; that is, why "first...second.. and at last but not least" is more preferred in English essays. Thus we see many samples in this study, though forming like the "general-particular" pattern, argue with details or sub-topics that are not parallel to each other and bearing no particularity to the general idea, which according to Wang and $\mathrm{Li}$ is called the mixedtype. Or put it another way, mixed type is a kind of unsuccessful attempt to follow the native general-particular discourse pattern. Though the non-English-like discourse pattern takes up more than $70 \%$ of the total 51 passages, the variance between grades in each discourse pattern renders the possible solution to the problem. As we can see from the figure below, there exist a tendency about discourse pattern distribution among the three groups [5]. As the grade increases, more and more students would like to adopt the general-particular pattern or at least mixed type of discourse development and less would like to employ the implicit pattern, which is to say as their English study goes on, it is possible for students to better realize the English-like feature in discourse development either totally or superficially, and some good try will come along as well.

\section{Conclusion}

This paper aims at exploring the cross-linguistic influence on Chinese students ESL writing on discourse level. Referring to the language transfer theory and contrastive rhetoric approach, this research finds its general hypothesis on cognitive ground that the previous acquired writing convention and rhetoric in the first language may serve as a procedure constrain on the production of second language. Then the paper compares the Chinese and English languages on discourse level based on previous studies and 6 research questions are put forward with their justification 
concerning three specific dimensions (thesis statement; discourse developing pattern and argumentation mode) and the extent of cross-linguistic influence (the variance between students of different English proficiency).

\section{Acknowledgment}

Fund Project: This article is an empirical study on the impact of Hoey's problem-solving discourse model on college students' English writing in the Hunan Provincial Philosophy and Social Science Fund Project (No. 11WLH11).

\section{References}

[1] Cai Jigang. The Differences in the Position of the Expression of English and Chinese Articles and Their Influence on Chinese Students' English Writing [J]. Foreign Language Teaching in Foreign Countries. 2007 (01)

[2] Shi Yizhi. The Anti-evidence of Chomsky's "Universal Grammar" Hypothesis - Inspiration from Cognitive Psychology [J]. Journal of PLA University of Foreign Languages. 2005 (01)

[3] Chen Suyan. Chinese Transfer in English Writing and Its Cultural Tracing [J]. Journal of Zhejiang Wanli University. 2004 (03)

[4] Wang Wenyu, Wen Qiufang. Native Language Thinking and Second Language Writing: A Study of College Students' English Writing Process [J]. Journal of PLA University of Foreign Languages. 2002 (04)

[5] Chen Zhonghua, Guan Xinping. The Cognitive Psychology Motivation of Macro Structure of Discourse [J]. Foreign Language Studies. 1999 (04) 